Chemotherapy 1978;24:1

\title{
To Our Readers
}

Beginning with this volume Chemotherapy is published in a two-column format, a space conserving design which permits the inclusion of more material in fewer pages. Although these volumes may feel slimmer than in the past, their actual contents have expanded.

We hope that this new format renders

Chemotherapy easier for you to read and handle and that you will appreciate these qualities as much as you will benefit from the increased amount of material presented. Authors may consider that due to the two-column presentation the number of printed pages free of charge is now six (approximately 12 type-written pages.) 